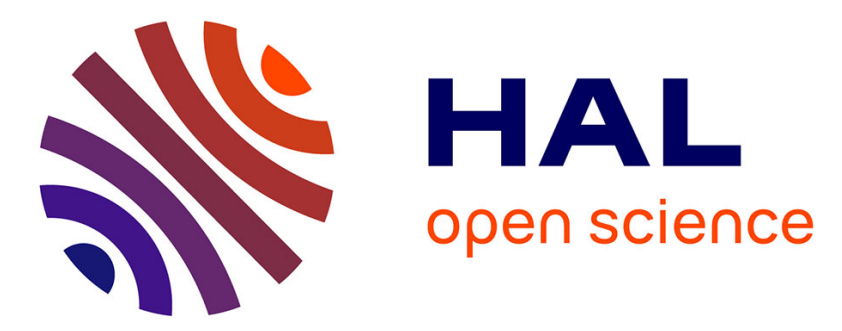

\title{
De la Responsabilité sociale des organisations à l'achat public responsable : entre contraintes et performances. Compte rendu de journée d'étude Olivier Gayot
}

\section{- To cite this version:}

Olivier Gayot. De la Responsabilité sociale des organisations à l'achat public responsable : entre contraintes et performances. Compte rendu de journée d'étude. Développement durable et territoires, 2019, Développement durable et territoires, 10 (1), 10.4000/developpementdurable.13666 . hal-02900761

\section{HAL Id: hal-02900761 \\ https://hal.univ-lille.fr/hal-02900761}

Submitted on 16 Jul 2020

HAL is a multi-disciplinary open access archive for the deposit and dissemination of scientific research documents, whether they are published or not. The documents may come from teaching and research institutions in France or abroad, or from public or private research centers.
L'archive ouverte pluridisciplinaire HAL, est destinée au dépôt et à la diffusion de documents scientifiques de niveau recherche, publiés ou non, émanant des établissements d'enseignement et de recherche français ou étrangers, des laboratoires publics ou privés.

\section{(1)(1) $\$(0)$}

Distributed under a Creative Commons Attribution - NonCommercial - ShareAlikel 4.0 


\section{Développement durable et territoires}

\section{De la Responsabilité sociale des organisations à} l'achat public responsable : entre contraintes et performances. Compte rendu de journée d'étude

From Social Responsibility of Organizations to fair public procurement : between constraints and performances. Report of a study day

\section{Olivier Gayot}

\section{OpenEdition \\ Journals}

Édition électronique

URL : http://journals.openedition.org/developpementdurable/13666

DOI : 10.4000/developpementdurable.13666

ISSN : 1772-9971

Éditeur

Association DD\&T

Ce document vous est offert par Université Lille 1

\section{Université de Lille}

Référence électronique

Olivier Gayot, « De la Responsabilité sociale des organisations à l'achat public responsable : entre contraintes et performances. Compte rendu de journée d'étude ", Développement durable et territoires [En ligne], Vol. 10, $n^{\circ} 1$ | Avril 2019, mis en ligne le 04 avril 2019, consulté le 01 juillet 2020. URL : http:// journals.openedition.org/developpementdurable/13666; DOI : https://doi.org/10.4000/ developpementdurable.13666

Ce document a été généré automatiquement le 1 juillet 2020. 


\section{De la Responsabilité sociale des organisations à l'achat public responsable : entre contraintes et performances. Compte rendu de journée d'étude}

From Social Responsibility of Organizations to fair public procurement : between constraints and performances. Report of a study day

\section{Olivier Gayot}

1 La journée d'étude «RSO et achats publics: entre contraintes et performances » s'est déroulée à Lille dans les locaux de la Maison européenne des sciences de l'homme et de la société (MESHS), le 23 novembre 2017. Elle a été organisée dans le cadre d'un projet de recherche pluridisciplinaire cofinancé par la Région des Hauts-de-France au titre du programme «Chercheurs-citoyens ». Le projet RSECO, «Une Responsabilité sociétale des entreprises repensée - de l'implication des acteurs à une réappropriation des politiques publiques ", s'est déroulé de janvier 2016 à janvier 2019. Il a été réalisé en partenariat avec une équipe de recherche pluridisciplinaire et l'association Yamana ${ }^{1}$. Le projet a été coordonné par Malik Bozzo-Rey (professeur en éthique à l'Institut catholique de Lille), avec la participation de Nicolas Postel et Richard Sobel (professeurs en économie à l'université de Lille), Guillaume Delalieux (maître de conférences en sciences de gestion à Sciences Po Lille), Mathias Amilhat (maître de conférences en droit public à l'université de Lille), Leslie Carnoye (post-doctorante dans le projet RSECO) et Frédéric Fournier (chef de projet à l'association Yamana). L'auteur du présent article a également participé au projet dans le cadre d'un stage de fin d'études de six mois de février à juillet 2018. L'équipe du projet RSECO s'est interrogée sur la mise en place d'initiatives de « Responsabilité sociétale des entreprises » (RSE) par les acteurs publics au niveau local ou régional, ainsi que sur le développement de l'achat public responsable (APR), en tant 
qu'outil de politique publique, d'un point de vue pluridisciplinaire. Cette journée a réuni une petite vingtaine de chercheurs, de professionnels et d'étudiants.

Trois temps ont rythmé les échanges. Tout d'abord, les interventions de Gérard Brunaud (ancien chargé de mission interministériel «Politique d'achats responsables", cofondateur et ancien secrétaire général de l'ObsAR $^{2}$ et membre de la Plateforme nationale pour la $\mathrm{RSE}^{3}$ ) et de Mathias Amilhat (maître de conférences en droit public à l'université de Lille) ont permis de mieux définir ce que sont les achats publics responsables et ont retracé dans les grandes lignes leur histoire, d'un point de vue institutionnel et juridique, en France. Dans un second temps, Yannick Leroy (acheteuseréférente APR à la communauté urbaine de Dunkerque (CUD), animatrice du réseau achat responsable du Nord-Pas de Calais) et Céline Frahtia-Levoir (cheffe du bureau supports et techniques d'achat à la direction des finances et des achats à la mairie de Paris) ont apporté leurs témoignages sur les formes que prend aujourd'hui la mise en place de politiques d'achats responsables dans leurs institutions respectives. Enfin, Nicolas Postel (professeur d'économie à l'université de Lille et ancien vice-président de l'université de Lille 1) a animé un temps de débat avec la salle et l'ensemble des intervenants, fort de ses activités de recherche autour de la RSE et de son expérience de gestion au sein de l'université de Lille 1.

3 En introduction, Leslie Carnoye a rapidement présenté le projet RSECO. Cette dernière a précisé qu'à l'origine l'ambition du projet était de réussir à mieux cerner les formes que pouvaient prendre les différentes démarches de RSE engagées par les collectivités territoriales. Assez rapidement, l'équipe a pu identifier que l'APR constituait un levier de transmission majeur pour les collectivités, au-delà des dispositifs managériaux internes comme les plans de promotion de l'égalité femmes-hommes, de la diversité ou des thématiques comme le bonheur au travail par exemple, qui peuvent faire l'objet de démarches labellisées «RSE » dans les collectivités territoriales. En effet, l'intérêt de l'entrée par l'APR est que ces derniers ont une véritable portée territoriale. La Commission européenne estime que la commande publique ${ }^{4}$ représentait dans l'Union européenne en 2017 environ 14 \% du PIB (Commission européenne, 2017). Le mouvement général d'externalisation des services publics qu'ont connu la plupart des pays européens depuis la fin des années 1970 peut expliquer le poids économique important de la commande publique européenne. Dès lors, l'APR peut aujourd'hui être compris comme un levier de politique publique essentiel, quand bien même il se caractérise par une ambiguïté fondamentale: son poids économique (et politique) grandit avec celui de l'externalisation des services publics.

\section{Histoire institutionnelle et juridique de l'APR en France : que permettent les nouveaux textes?}

4 Un marché public est « un contrat administratif conclu à titre onéreux entre un acheteur et un opérateur économique pour répondre aux besoins de l'acheteur en matière de travaux, de fournitures ou de services. Les marchés qui répondent à un besoin dont la valeur estimée est égale ou supérieure à $25000 €$ euros hors taxe (HT) doivent être conclus par écrit " ("Définition et principes d'un marché public »). La législation européenne a ainsi imposé le respect de trois grands principes de concurrence: la liberté d'accès à la commande publique, l'égalité de traitement entre les candidats et la transparence. 
Durant son intervention, Gérard Brunaud est revenu sur la diffusion progressive de pratiques d'APR dans les collectivités territoriales en France. Dans les années 1990, c'est au travers de la mise en place de clauses sociales d'insertion que se sont développés les APR dans plusieurs villes (à Strasbourg notamment), ainsi que dans quelques écoles de commerce et entreprises publiques (La Poste, la RATP, la SNCF...). Gérard Brunaud a également précisé le contexte dans lequel l'État l'a sollicité pour engager, en 2007, une mission interministérielle relative à l'amélioration des pratiques d'achats de l'État. Cette mission, demandée par Jean-Baptiste de Foucauld ${ }^{5}$, a été initiée à un moment où l'État incitait les collectivités territoriales à introduire des clauses sociales ou environnementales dans leurs marchés et entendait montrer l'exemple dans ses propres marchés. Gérard Brunaud a aussi souligné que l'Union européenne, bien qu'ayant institué les principes de concurrence qui cadrent aujourd'hui l'achat public, promouvait l'APR depuis longtemps. La CEE (Communauté économique européenne) a en effet lancé le programme "Green Public Procurement» au début des années 1990. L'objectif de ces achats publics « verts » » était que $50 \%$ des marchés publics soient dotés d'une clause environnementale (ce qui n'est toujours pas le cas aujourd'hui).

Gérard Brunaud s'est montré plutôt optimiste quant à l'institutionnalisation progressive de l'APR en France. Selon lui (et ce point est également fortement mis en avant par l'ObsAR), l'APR a l'intérêt de permettre aux collectivités de passer d'une logique d'achat basée sur les prix à court terme à une réflexion en termes de coût global. Cela contribue à renforcer la prise en compte du long terme, essentielle à une gestion "efficace » des deniers publics, dans les marchés publics. Enfin, Gérard Brunaud a noté le manque d'expertise de certaines collectivités (notamment les plus petites) et a vivement déploré le caractère peu restrictif du Spapser ${ }^{6}$ qui ne concerne que les collectivités disposant de plus de 100 millions d'euros de budget annuel pour leurs achats.

7 Dans un second temps, Mathias Amilhat a insisté sur le fait que les règles de droit ne doivent pas être lues comme des règles neutres, mais qu'elles sont au contraire liées à une idéologie. L'histoire juridique de l'achat public en est selon lui une bonne illustration. Le tout premier code des marchés publics est daté de 1964 et le droit public a connu une période de relative stabilité textuelle jusqu'en 2001. À partir de 2001, la France a connu un fort développement dans ses ordonnances et leurs décrets d'application, en lien avec la construction du droit de l'Union européenne. En 2004 et 2006, deux nouveaux codes des marchés ont été produits. En 2014, la révision de la directive européenne sur les achats publics $^{7}$ transcrite en loi nationale ${ }^{8}$ ainsi que son décret d'application ${ }^{9}$ ont abrogé le code des marchés français. Dès lors, un certain nombre de dispositifs favorables au développement de pratiques d'achats plus responsables ont été consolidés (sourcing ${ }^{10}$, allotissement ${ }^{11}$, analyse du cycle de vie, etc.).

8 Toutefois, selon Mathis Amilhat, la prise en compte effective de considérations sociales et environnementales est restée très limitée, au regard de l'interprétation (restrictive) que propose la Cour de justice européenne des différents textes. Cependant, ces textes admettent que l'achat public peut être mis au service d'objectifs sociaux et environnementaux et ils rendent même obligatoire la prise en compte du développement durable dans les décisions d'achat. C'est une transformation profonde de la nature du droit de la commande publique, car ce droit "pratique» et contingent passe d'une application strictement juridique, dont l'unique objectif est de garantir la sécurité des contrats, à un nouvel outil de régulation économique (Cantillon, 2010). Néanmoins, 
l'ampleur du changement que cela représente peut être, selon lui, relativisée, car les principes de concurrence des marchés publics priment toujours sur les autres critères.

Mathias Amilhat a conclu en soulignant la difficulté de mobiliser la commande publique comme nouvel outil de régulation au service d'objectifs de politiques économiques. L'intervenant a également souligné qu'en l'absence de jurisprudence autour des nouveaux textes, le choix de casser un marché revient à l'appréciation du juge, et celui-ci peut alors favoriser les principes de concurrence, ou bien les critères de développement durable. Pour le moment, on ne peut qu'espérer une approche plus souple des marchés publics.

\section{Pratiques et innovations autour de l'APR : les exemples de la Communauté urbaine de Dunkerque (CUD) et de la Ville de Paris}

10 La deuxième partie de l'après-midi a été consacrée aux retours d'expériences des praticiens de la commande publique, autour des témoignages de la Communauté urbaine de Dunkerque (CUD) et de la Ville de Paris.

11 Yannick Leroy a présenté l'historique des actions menées par la CUD depuis son arrivée en 2002. Celle-ci a indiqué avoir commencé à s'intéresser aux APR dès les années 2000, en lien avec la mise en place de l'Agenda 21 au niveau local. Ce sont ensuite deux expériences d'achats durables "malheureux $»^{12}$ qui ont poussé la CUD à mieux spécifier les critères techniques de ses achats. Plusieurs démarches ont alors été engagées, qui ont donné lieu à la création d'un guide sur les achats éthiques en 2004 et à la mise en place de critères d'achat éthique sur le bois, en 2006. En 2016 et jusqu'à aujourd'hui, la CUD continue de développer ses pratiques d'achats responsables de manière volontariste, car l'agglomération n'est pas soumise à la mise en place d'un Spapser ${ }^{13}$. Trois actions phares peuvent être mentionnées : le développement de l'analyse de produits en termes de cycle de vie, en travaillant sur la précision des spécifications techniques; l'insertion de clauses sociales dans différents marchés (notamment la blanchisserie et les petits travaux de nettoyage), grâce au travail de facilitateurs mettant en lien la collectivité et les entreprises de réinsertion sur le territoire; et la mise en place d'une nomenclature dédiée pour les marchés réservés dans la blanchisserie, l'achat de grosserie, le nettoyage de petits locaux ou la mise sous pli, qui permettent à la collectivité de s'adresser directement aux entreprises du territoire dans le secteur du handicap.

Selon Yannick Leroy, il est important que la commande publique soit perçue comme un levier pour le développement de l'économie circulaire, de l'achat local et de la performance énergétique. L'APR est un outil clé pour favoriser les échanges entre la collectivité et les entreprises du territoire en ce qu'il permet d'éviter le greenwashing, de favoriser le sourcing et de simplifier l'accès aux marchés publics pour les PME du territoire. Toutefois, l'une des difficultés concerne le rôle et la place des indicateurs dans les APR. Pour Yannick Leroy, il est nécessaire de réussir à évaluer non seulement la part des achats responsables dans les achats totaux de la collectivité, mais aussi l'impact sur le territoire des pratiques d'achats responsables.

13 Par la suite, Céline Frahtia-Levoir a présenté les grandes étapes de la mise en place des APR à la Ville de Paris. Les démarches de développement durable ont aussi été instaurées dès le début des années 2000. Plus spécifiquement, entre 2003 et 2009, la Ville a développé 
ses premières clauses d'insertion et ses clauses environnementales. À partir de 2009, jusqu'en 2014, la Ville de Paris a regroupé ses acheteurs et fusionné la direction des achats et celle des finances pour n'en former qu'une seule : la Direction des finances et des achats (DFA).

La Ville de Paris dispose aujourd'hui d'un budget total compris entre 1,1 et 1,6 milliard d'euros. $80 \%$ du budget est géré par la DFA et les $20 \%$ restants sont gérés au sein des 20 directions prescriptrices. La DFA compte 200 collaborateurs, y compris le BSTA dirigé par Céline Frahtia-Levoir. Ce dernier a été créé pour veiller à la prise en compte des critères de développement durable dans les marchés, et il joue le rôle de conseiller auprès des acheteurs dans les différentes directions, ainsi qu'auprès du Conseil de Paris.

Céline Frahtia-Levoir a insisté en conclusion sur l'importance fondamentale du sourcing. La Ville de Paris a ainsi mis l'accent sur cette pratique depuis 2009, le but étant de faire coïncider au mieux l'offre des fournisseurs et la demande de l'acheteur public, tout en permettant de prendre en compte les améliorations et les innovations du milieu fournisseur, faciliter la capacité de réponse des fournisseurs, fournir des prestations de service de meilleure qualité, réaliser des économies d'achat sur le long terme, prendre en compte le commerce équitable dans les achats et faciliter l'accès aux PME à l'aide de l'allotissement. Cette pratique demande toutefois des moyens humains, financiers et matériels importants, car cela implique de pouvoir fortement anticiper sur la passation des marchés.

\section{L'APR en débat : des acheteurs publics soumis à des injonctions contradictoires?}

Le troisième et dernier temps de l'après-midi a été consacré au débat, introduit par Nicolas Postel. Ce dernier a soulevé plusieurs questions mettant en évidence des contradictions qui pourraient contrevenir à la dynamique d'institutionnalisation de l'APR. Comment répondre au surcoût lié à la montée en qualité des APR dans un contexte de restriction très importante des dépenses publiques? Comment mettre en place des démarches de sourcing, qui impliquent de développer des relations quasi " personnelles" avec à la fois les entreprises, le groupement de commande et la mutualisation ? Comment concilier la volonté de simplification législative, avec la montée en technicité des critères et des démarches d'APR ?

Sur la question du surcoût, Yannick Leroy a indiqué que ce dernier est bien moins important aujourd'hui qu'au début des démarches d'APR, il y a dix ans. D'autre part, selon elle, au-delà du contexte budgétaire tendu, il est nécessaire que les démarches d'achats responsables soient intégrées à la politique globale de l'organisation, afin que les comportements des agents publics et des citoyens puissent évoluer. Céline Frahtia-Levoir a quant à elle réagi en expliquant qu'il n'y avait pas forcément de contradiction entre l'APR et la diminution des dépenses publiques, car ces deux éléments obligent à reposer la question du besoin, parallèlement à celle du budget. Selon elle, si on raisonne en termes de coût global, sur le long terme, la montée en qualité et la réduction des coûts sont compatibles.

Concernant la contradiction potentielle entre le sourcing, les groupements de commandes et la mutualisation, Yannick Leroy a précisé que ces éléments peuvent en partie être conciliés. Selon elle, c'est à l'acheteur de connaître son territoire et de réaliser un achat 
intelligent. Ainsi, le groupement de commandes et la mutualisation ne sont pas toujours nécessaires, mais sont parfois utiles : quand les PME ne peuvent pas répondre à l'offre, ou pour les collectivités dont les capacités d'ingénierie sont plus modestes. L'acheteur doit réaliser un travail d'économiste sur son territoire et il serait essentiel que des formations professionnelles soient davantage mises en place pour l'y aider. Dans cette optique, Gérard Brunaud a souligné qu'il était très important de lutter contre la réticence des centres de formation à les mettre en place, car ils ne remplacent pas les chargés de projet et les grandes structures ne portent pas encore ces formations, car elles n'en ont pas encore vu l'intérêt.

Le sourcing a également été une question très discutée, mais le point essentiel à retenir est celui de Mathias Amilhat qui a expliqué que le sourcing reste une pratique vis-à-vis de laquelle les acheteurs doivent se montrer prudents, compte tenu du cadre fixé par le droit. Si un concurrent sur un marché peut prouver qu'il n'a pas été mis au courant du sourcing, l'acheteur s'expose à un risque pénal. Nicolas Postel a alors posé la question du recrutement de nouveaux acheteurs pour coïncider avec la montée en technicité des marchés. Gérard Brunaud et Yannick Leroy ont répondu en insistant sur le besoin de développer la formation des acheteurs, et Céline Frahtia-Levoir a défendu la nécessité pour l'acheteur de développer des outils plus performants et une capacité de créativité.

En somme, la journée d'étude «RSO et achats publics: entre contraintes et performances " a permis de montrer que le droit et les pratiques des acheteurs en matière de commande publique connaissent aujourd'hui une période de transformations importantes. Le développement durable semble de mieux en mieux intégré, en tant qu'objectif de politique publique, à la fois dans les textes juridiques et dans les pratiques des acteurs territoriaux. L'Union européenne joue aujourd'hui un rôle moteur pour le développement de l'APR qu'elle considère désormais comme un levier stratégique essentiel, notamment dans la perspective d'une croissance «verte, intelligente et innovante » (Commission européenne, 2010). Toutefois, les principes de concurrence semblent rester prioritaires. Pour l'heure, la jurisprudence autour des nouveaux textes est très attendue et elle permettra de mieux cerner l'ampleur du changement de vision en cours autour de l'achat public et les nouvelles marges de manœuvre effectives des acheteurs publics.

\section{BIBLIOGRAPHIE}

Cantillon G., 2010, « L'achat public durable, un outil au service de l'État régulateur », Revue française d'administration publique, $\mathrm{n}^{\circ}$ 134, p. 335-350., https://doi.org/10.3917/rfap.134.0335.

Commission européenne, 2010, «Europe 2020 : A strategy for a smart, sustainable and growth », Bruxelles, Commission européenne.

Commission européenne, 2017, 3 octobre, « Making Public Procurement work in and for Europe. Définition et principes d'un marché public », https://www.service-public.fr/professionnelsentreprises/vosdroits/F33466, consulté 20 novembre 2018. 
OCDE, 2011, « Government at a Glance 2011 », https://www.oecd-ilibrary.org/content/ publication/gov_glance-2011-en.

OCDE, 2017, Government at a glance, OCDE publishing.

\section{NOTES}

1. Yamana est une ONG de conseil en stratégie RSE. Ses domaines d'expertise sur les achats responsables concernent principalement le secteur textile et les cosmétiques. Elle a également réalisé plusieurs missions d'assistance à maîtrise d'ouvrage auprès de plusieurs collectivités territoriales. Plus d'informations sur l'association peuvent être trouvées ici: http:// www.yamana-mvd.org/.

2. L'Observatoire des achats responsables (ObsAR) est une association visant à promouvoir les pratiques d'achats responsables auprès des entreprises privées et des pouvoirs publics. Elle réalise régulièrement des enquêtes publiées sous la forme d'un baromètre des achats responsables. Plus d'informations peuvent être trouvées ici : http://www.obsar.asso.fr/.

3. La plateforme nationale pour la RSE est une instance de concertation créée par le Premier ministre sous l'impulsion de plusieurs parties prenantes le 16 septembre 2012 et installée au sein de France Stratégie en juin 2013. Plus d'informations peuvent être trouvées ici: http:// www.strategie.gouv.fr/chantiers/plateforme-rse.

4. La commande publique réfère à l'achat de bien, de services et de travail par les gouvernements et les entreprises publiques (OCDE, 2017). Le terme de gouvernement comprend l'État, les collectivités territoriales et la sécurité sociale.

5. Jean-Baptiste de Foucauld est un haut fonctionnaire français, ancien commissaire au plan (1992-1995), membre du Conseil d'orientation pour l'emploi, du Conseil national de l'information statistique, et fondateur, administrateur, parrain ou membre de nombreuses associations (Solidarités nouvelles face au chômage, Coordination pour l'éducation à la non-violence et à la paix...).

6. La mise en place d'un Schéma promotionnel des achats publics socialement et écologiquement responsables (Spapser) est devenue une obligation légale depuis la loi sur l'Économie sociale et solidaire de 2014 et la loi sur la Transition Energétique de 2015, mais seulement pour les collectivités de taille importante.

7. Directive 2014/24/UE du 26 février 2014.

8. Ordonnance $n^{\circ} 2015-899$ du 23 juillet 2015 relative aux marchés publics.

9. Décret $\mathrm{n}^{\circ}$ 2016-360 du 25 mars 2016 relatif aux marchés public.

10. Le sourcing découle du verbe anglais To source et fait référence, dans le cadre des marchés publics, au fait d'identifier les fournisseurs potentiels pour répondre à un besoin exprimé par la collectivité.

11. L'allotissement est le découpage d'un marché principal en différents marchés de plus petite taille, appelés « lots », pouvant être attribués à des prestataires différents. Des entreprises qui n'auraient pas pu répondre au marché principal à cause de leur taille modeste peuvent dès lors candidater.

12. Un achat dans le domaine des produits d'entretiens écoresponsables infructueux, et un achat de papier recyclé qui s'est révélé de très mauvaise qualité.

13. Le budget achat de la CUD s'élève à 88 millions d'euros HT, selon Yannick Leroy. 


\section{RÉSUMÉS}

L'achat public responsable peut être compris comme une nouvelle conception de l'achat public, qui pourrait permettre de réaffirmer le rôle des acteurs publics dans l'institutionnalisation du développement durable, notamment à l'échelle des territoires. L'achat public responsable étant une notion récente, il n'existe aujourd'hui quasiment aucune littérature académique en économie sur le sujet. L'objectif du présent article est d'exposer différents points de vue pratiques et théoriques autour de l'achat public responsable, sans prétendre à une forme d'exhaustivité. Ces différentes perspectives ont été abordées durant la journée d'étude «RSO et achats publics, entre contraintes et performances » ayant eu lieu le 23 novembre 2017 à la MESHS (Lille) organisée dans le cadre du projet «Une RSE repensée - de l'implication des acteurs à une réappropriation des politiques publiques » (RSECO).

Responsible public procurement can be understood as a new conception of public procurement, which could reaffirm the role of public actors in the institutionalization of sustainable development, especially on a territorial scale. There is nearly no academic literature in economics about responsible public procurement, as the concept is rather recent. The point of this article is to present several practical and theoretical perspectives around responsible public procurement, not pretending to any form of exhaustiveness. These perspectives were discussed during a study day organized within the RSECO project «CSR and public procurement, between constraints and performances » which took place on November $23^{\text {rd }}, 2017$ at the MESHS (Lille, France).

\section{INDEX}

Mots-clés : achat public responsable, responsabilité sociétale des organisations, collectivité territoriale, développement durable

Keywords : public procurement, social responsibility of organizations, regional government, sustainable development

\section{AUTEUR}

\section{OLIVIER GAYOT}

Olivier Gayot réalise un doctorat en économie relatif à la question des achats publics responsables dans la région des Hauts-de-France au sein du laboratoire Clersé (UMR 8019). 